\title{
Easing the transition to residency through urology bootcamp
}

\author{
Raees Cassim, $M D^{1}$; Edward D. Matsumoto, MEd, $M D^{1,2}$ \\ 'Department of Surgery, Division of Urology, McMaster University, Hamilton, ON, Canada; 2 St. Joseph's Healthcare Hamilton, McMaster Institute of Urology, Hamilton, ON, Canada
}

Cite as: Cassim R, Matsumoto ED. Easing the transition to residency through urology bootcamp. Can Urol Assoc J 2021;15(4):107. http://dx.doi.org/10.5489/cuaj.6994

See related paper on page 98

$\mathrm{D}$ espite the support of residency programs, the transition from medical student to resident was harsh. Add a once-in-a-generation pandemic, and it made it almost impossible. For many, starting residency meant moving to a new city and learning the ins and outs of a new hospital. With COVID upon us, gone are residents' socials and other opportunities to meet first-year residents outside of our respective programs to help ease the social isolation. Furthermore, for many of us, medical school ended quite abruptly, limiting critical clinical encounters to augment our learning and prepare us for residency.

The new responsibility of being a physician rather than a medical student also comes cursed with self-doubt. As a resident, I find myself questioning my actions, as they carry the weight of responsibility. While still supervised, the opportunity to cause harm through mistakes is sobering. Gaining confidence that I have the knowledge and skillset to manage the many on-call emergencies was key to feeling comfortable in my new role as a surgical resident. Typically, this confidence came through clinical experience; however, simulation and teaching have a role to play as well.

The urology bootcamp was a tool that helped ease the transition to residency and specifically my approach to oncall issues. ${ }^{1}$ Unlike typical lectures, which tend to focus on minutia, the didactic sessions were centered on clinical pearls of assessing and managing urological emergencies. The hands-on sessions provided us with a familiarity of the basic urological tools we may need to use overnight. While the bootcamp is no substitute for experience, it provides a framework to approach common problems. This way, we have the means to recognize critical patients, ask key questions, and order appropriate investigations to provide the key information to our seniors or attendings when reviewing our patients. Furthermore, simulation, while not the same as true operative exposure, provides us with the opportunity to develop the fundamental technical skills while avoiding accidental harm to patients and the time constraints of the operating room. At the very least, it allows us to answer the inevitable, "What do you want to do next?" question from our attendings.

In addition to the urology bootcamp, I also had the opportunity to participate in the Surgical Foundations bootcamp. While this provided some basic knowledge to manage a postoperative patient, it was not overly relevant to our specialty. With most residents starting on service as a result of competency-based education, the opportunity to participate in a tailored bootcamp is a necessity.

The best way to describe the relevance of the urology bootcamp is to describe my first weekend on-call. In one weekend, I experienced multiple septic stones, a query torsion, a difficult Foley, and a priapism. The bootcamp taught me the key features to looks for when determining if patients should go to the operating room or had time for further evaluation. The cystoscopic catheter insertion simulator gave me the skill set to manage a difficult catheter without requiring my senior to scrub in. Did the bootcamp make my first call easy? No, but it made it less stressful.

There is another, less-recognized benefit to the urology bootcamp; it helps strengthen the urological community. While only two schools participated in the bootcamp, it still provided the opportunity to reunite with friends made during our electives. In the era of social distancing, this was a muchwelcomed opportunity. Overall, the bootcamp experience made me feel comfortable being thrown back into clinical duties. I would highly recommend bootcamp participation to future residents and look forward to the opportunity to lead bootcamp sessions in the future.

Competing interests: The authors report no competing personal or financial interests related to this work.

\section{References}

1. Wang Y, Hoogenes J, Clark R, et al. Development, implementation, and evaluation of a competency-based didactic and simulation-focused boot camp for incoming urology residents: Report of the first three years. Can Urol Assoc J 2021;15:98-105. hitp://dx.doi.org/10.5489/cuai.6679

Correspondence: Dr. Raees Cassim, Division of Urology, Department of Surgery, McMaster University, Hamilton, 0N, Canada; raees.cassim@medportal.ca 